\title{
Optical Effects of Spacecraft-Environment Interaction: Spectrometric Observations of the DE-2 Satellite
}

\author{
Vincent J. Abreu, ${ }^{*}$ Wilbert R. Skinner, $\dagger$ and Paul B. Hays $\ddagger$ \\ University of Michigan, Ann Arbor, Michigan \\ and \\ Jen-Hwa Yee§ \\ Harvard-Smithsonian Center for Astrophysics, Cambridge, Massachusetts
}

\begin{abstract}
Data from the Fabry-Perot interferometer on board the Dynamics Explorer-2 (DE-2) satellite are used to show that the contaminant glow observed by Yee and Abreu at $7320 \AA$ on the Atmosphere Explorer (AE) satellites is produced by emission lines of one or more species. A comparison is made of the contaminant spectrum near $7320 \AA$ with the nightglow $O H$ spectrum measured below $155 \mathrm{~km}$. Evidence is presented to the effect that $\mathrm{OH}$ might be one of the metastable species producing the glow.
\end{abstract}

\section{Introduction}

$\mathbf{T}_{\mathrm{s}}$ HE characteristics and spectral variation of the optical glow induced by spacecraft-atmosphere interaction have been recently described by Yee and Abreu ${ }^{1,2}$ using photometric data obtained by the Visible Airglow Experiment (VAE) on board the Atmosphere Explorer satellites. ${ }^{3}$ Their data showed that 1) the most intense radiation comes from surfaces facing the direction of motion of the satellite; 2) the contamination has a band or continuum spectrum which is brighter toward the red; and 3) there is a strong correlation between emission intensity and oxygen atom density in the $160-280-\mathrm{km}$ altitude range. Yee and $\mathrm{Abreu}^{1}$ suggested that the glow is produced by molecules which are formed and ejected from the satellite surface in a metastable state after surface particles undergo chemical reaction or direct impact collisions with incoming $\left.\mathrm{O}^{3} \mathrm{P}\right)$ atoms at the satellite velocity $(\sim 8$ $\mathrm{km} / \mathrm{s}^{-1}$ ). Later, based on the apparent spectral distribution and the radiative lifetime deduced by Yee and Abreu ${ }^{1}(\sim 5$ $\mathrm{ms})$, Slanger ${ }^{4}$ showed that the $\mathrm{OH}$ Meinel bands are a tenable identification for the AE satellite glow. He proposed that $\mathrm{OH}$ is produced by the interaction of $5-\mathrm{eV} \mathrm{O}\left({ }^{3} \mathrm{P}\right)$ atoms and adsorbed water and/or C-H bonds in the satellite construction material. So far, however, no spectroscopic measurements have been reported which confirm this hypothesis.

The Dynamic Explorer-2 spacecraft is a modified version of the Atmosphere Explorer spacecrafts, whose payload included a high-resolution Fabry-Perot interferometer (FPI) designed to measure temperatures and winds in the thermosphere. Given the similarity between the spacecrafts, we have used high-resolution data obtained by the FPI in a spectral region centered around $7320 \AA$ in order to provide further evidence concerning the nature of the processes producing the optical glow. The spectral region to be investigated corresponds to the 8-3 $\mathrm{OH}$ emission band. This emission is also very strong in the terrestrial nightglow producing a narrow emission layer which peaks in the upper mesosphere. Consequently, a comparison of spectra obtained

Presented as Paper 83-2657 at the AIAA Shuttle Environment and Operations Conference, Washington, D.C., Oct. 31-Nov. 2, 1983; submitted Jan. 6, 1984; revision received April 2, 1984. Copyright (C) American Institute of Aeronautics and Astronautics, Inc., 1984. All rights reserved.

*Associate Research Scientist, Space Physics Research Laboratory.

†Assistant Research Scientist, Space Physics Research Laboratory.

$\ddagger$ Professor, Space Physics Research Laboratory.

$\S$ Research Associate. at mesospheric heights with those obtained at satellite altitudes $(\sim 250 \mathrm{~km})$ should provide the necessary information to show whether $\mathrm{OH}$ is one of the emitting metastable species producing the optical glow. This analysis is presented in this paper.

\section{Description of Instrument}

The Fabry-Perot interferometer on Dynamics Explorer-2 is a remote sensing instrument designed mainly to measure the temperature, meridional wind, and density of metastable $O\left({ }^{1} \mathrm{~S}\right)$ and $O\left({ }^{1} \mathrm{D}\right)$ atoms and the $\mathrm{O}^{+}\left({ }^{2} \mathrm{P}\right)$ ion in the thermosphere. A detailed description of the instrument has been given by Hays et al., ${ }^{5}$ Killeen et al., ${ }^{6}$ and Killeen and Hays. ${ }^{7}$

The measurements are made with a high resolution FabryPerot etalon, which performs a wavelength analysis on light detected from atmospheric emission features by spatially scanning the interference fringe plane with 12 concentric ring detectors. The scan is linear in wavelength, covering a spectral range equal to $0.01796 \AA$ per detector channel at $7320 \AA$. The number of free spectral ranges focused on the detector is 1.0135 at this wavelength. The spectral region for analysis was selected by a $10 \AA$ halfwidth interference filter centered at $7320 \AA$. The etalon and detector parameters of interest are summarized in Table 1.

A sequential altitude scan performed by a commandable horizon scan mirror provides the spatial information at 16 tangent heights below the orbit of the satellite. Figure 1 is a schematic diagram which shows the detector position relative to the velocity vector of the spacecraft as well as the range of tangent altitudes scanned by the mirror. The altitude scan was limited to the angular region from 5 to $15 \mathrm{deg}$ below the horizon, with a field of view of $0.9 \mathrm{deg}$ (half-cone angle).

The sensitivity of the instrument was determined in a preflight calibration to be 0.04 counts/Rayleigh-s. For this analysis the sensitivity was also determined by an in-flight calibration using the daytime thermospheric $\mathrm{O}^{+}\left({ }^{2} \mathrm{P}\right)$ emission

Table 1 Parameters of interferometer

\begin{tabular}{ll}
\hline \hline Free spectral range, $\AA$ & $0.21265(7320)$ \\
$\begin{array}{l}\text { Fraction of the free spectral } \\
\text { range on detector, } \AA\end{array}$ & $1.0135(7320)$ \\
Detector anode structure & 12 concentric rings \\
& equal area anodes \\
Spectral range per anode, $\AA$ & $0.01796(7320)$ \\
Filter bandwidth, $\AA$ & $7320 \pm 10$ \\
\hline \hline
\end{tabular}




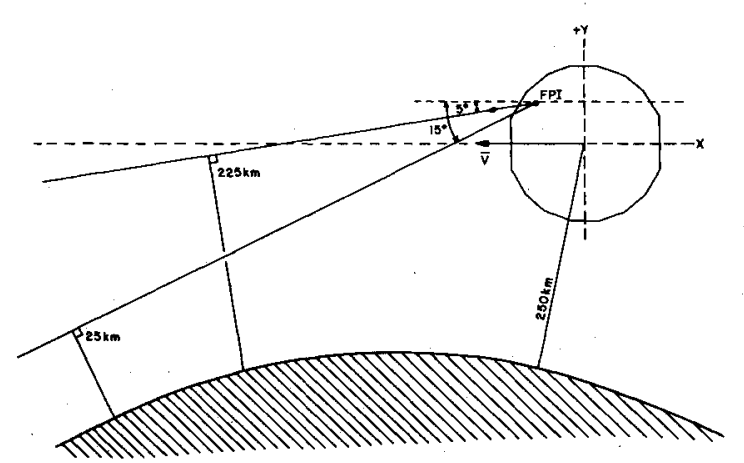

Fig. 1 Detector position relative to the velocity vector of the spacecraft and the range of tangent altitude scanned by the mirror.

at 7319.079 and $7320.154 \AA$. The production and loss processes, as well as the reaction rates involved in the calculation of the volume emission rate of this emission are well known. The sensitivity was then estimated from a theoretical calculation of the brightness ${ }^{8}$ and simultaneous measurements of the Fabry-Perot detector counts, the thermospheric temperature and the $\mathrm{O}$ and $\mathrm{N}_{2}$ densities made at the satellite altitude. Both calibrations are in good agreement.

For illustrative purposes, Fig. 2 shows a spectrum of the $\mathrm{O}^{+}\left({ }^{2} \mathrm{P}\right)$ emission at 7319.079 and $7320.154 \AA$. The units in the vertical axis are counts/integration period (I.P.). One integration period equals $0.22 \mathrm{~s}$. Since the transfer function of the Fabry-Perot is periodic, the relative position of the two lines in the image plane detector depends on $\Delta \lambda / \mathrm{FSR}$, where $\Delta \lambda$ is the wavelength separation between the two lines and FSR is the free spectral range of the etalon. The channel separation (number of channels apart) in the image plane detector is given by

$$
\left[\left(\frac{\Delta \lambda}{\mathrm{FSR}}-\mathrm{INT}(\Delta \lambda / \mathrm{FSR})\right)\right] \cdot \mathrm{FSR} / \delta \lambda
$$

where INT represents the integer part of the argument and $\delta \lambda$ is the spectral range of a ring. The doublet under consideration is 0.65 channels apart. Consequently, the two lines appear superimposed as a single line centered on channel 6 . The width of the line corresponds to a temperature of $\sim 1000 \mathrm{~K}$

\section{Data Analysis}

We have chosen for this study spectra measured by the FPI at tangent heights between 60 and $225 \mathrm{~km}$. These spectra were obtained while the spacecraft was near perigee $(-250 \mathrm{~km})$ and in a despun mode. Data were rejected if the solar zenith angle at the tangent point or at the satellite location was less than $130 \mathrm{deg}$ and if the absolute value of the magnetic latitude was greater than $55 \mathrm{deg}$ at the satellite location. If the satellite was moving poleward, data were rejected if the absolute value of the magnetic latitude was greater than $40 \mathrm{deg}$ at the tangent height. The above constraints ensured that data were not contaminated by daytime or high-latitude auroral emissions.

The most prominent features in the spectral range of interest $(7320 \AA \pm 10 \AA)$ in the night airglow are the vibrationrotation transitions in the ground electronic level of $\mathrm{OH}$. The excitation process for this emission is generally assigned to the reaction $^{9}$

$$
\mathrm{H}+\mathrm{O}_{3} \rightarrow \mathrm{OH}\left(\mathrm{X}^{2} \Pi, v\right)+\mathrm{O}_{2}
$$

In general, the $\mathrm{OH}$ emission occurs in a thin layer which peaks around $90 \mathrm{~km}$. The width of the layer is of the order of $10 \mathrm{~km}$ at half-intensity. ${ }^{10}$ Based on the morphology of the nightglow just presented, one would expect the observed spectra below $155 \mathrm{~km}$ to be that of $\mathrm{OH}$, while those above $155 \mathrm{~km}$, would be

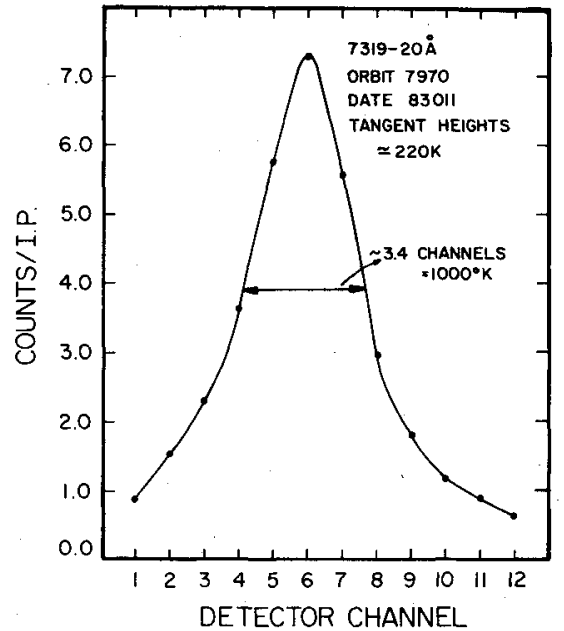

Fig. 2 Spectrum of the $\mathrm{O}^{+}\left({ }^{2} \mathrm{P}\right)$ emission obtained during the daytime.

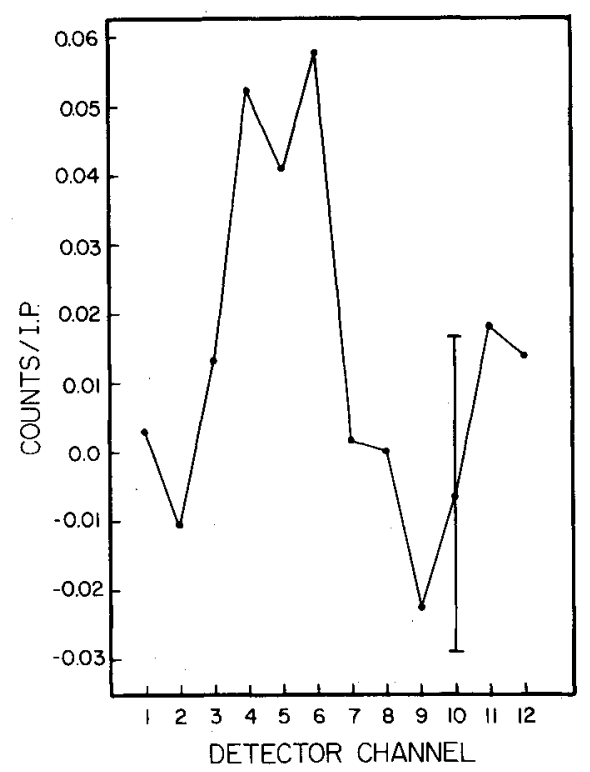

Fig. 3 Spectrum of the contaminant glow.

expected to be the spectra of the contaminant glow. This hypothesis will be investigated further in this analysis.

\section{Contaminant Glow Spectrum}

A spectrum of the emission above $155 \mathrm{~km}$ has been obtained by averaging approximately 6000 nighttime spectra with tangent heights up to $\sim 225 \mathrm{~km}$. The average satellite altitude was $253 \mathrm{~km}$. The spectrum is presented in Fig. 3. The spectra had an estimated dark count removed and were normalized to remove interchannel sensitivity differences. The negative counts shown in Fig. 3 are due to an imperfect knowledge of the dark count.

One may wonder if the observed spectrum is that of the contaminant glow or if it is instead the spectrum of the nightglow in the thermosphere. Evidence that it is the former is provided by the fact that the same spectral shape and intensitites are observed at all mirror positions with tangent heights above $155 \mathrm{~km}$. Further proof is provided by comparing the photometric intensity observed here with the 7319$20 \AA$ brightness of the glow observed at $250 \mathrm{~km}$ by Yee and Abreu ${ }^{1}$ using data from the VAE photometers on the Atmosphere Explorer satellites. The photometric brightness is obtained by adding the counts from each detector and multiplying the resulting counts by the calibration factor. The 
brightness thus obtained is -20 Rayleighs. This is in good agreement with the 7319-20 $\AA$ contaminant emission observed at $250 \mathrm{~km}$ by Yee and Abreu. ${ }^{1}$ Consequently, we conclude that the spectrum observed above $155 \mathrm{~km}$ by the Fabry-Perot interferometer is that of the contaminant glow, and that the photometric brightness observed by Yee and Abreu $^{1}$ on the AE satellites is not a continuum, but is produced by the emission lines of one of more species which remain to be identified. For this purpose we will next consider the $\mathrm{OH}$ spectra measured below $155 \mathrm{~km}$.

\section{OH Spectra}

Figure 4 shows spectra at different tangent heights obtained during orbit 8058 . The intensity of each spectrum as a function of altitude is consistent with the presence of a narrow layer which peaks at $\sim 80 \mathrm{~km}$. Below this altitude the spectra consist of two distinct emission lines which peak on channels 4 and 9 , respectively. Above $80 \mathrm{~km}$, the two lines come closer and seem to converge at approximately $92 \mathrm{~km}$. The apparent convergence of the two lines is due to a field-of-view effect, which comes about when the thin emission layer is viewed from above by the 12-channel detector. Under these circumstances, each channel in the detector has a different field of view. The different intensitites observed by the detector channels cause the distortion observed in the spectra. The distortions are greater in the outer channels of the detector. In order to correct the spectra it is necessary that the signal from each detector channel be deconvolved with the field of view. The large uncertainties in the data, however, limit the accuracy of the inversion process and the recovered spectra are not free of distortions. We have effected the deconvolution of spectra from tangent heights equal to 62,72 , and $82 \mathrm{~km}$ after normalizing them to their respective photometric brightnesses (area). The resultant spectrum is shown in Fig. 5. Spectra from the topside of the emission layer were not averaged because the effect of the field of view is most significant in that region. It should be noted that the separation between the two spectral lines observed is approximately five channels. Laboratory measurements of the 8-3 band of $\mathrm{OH}$ have

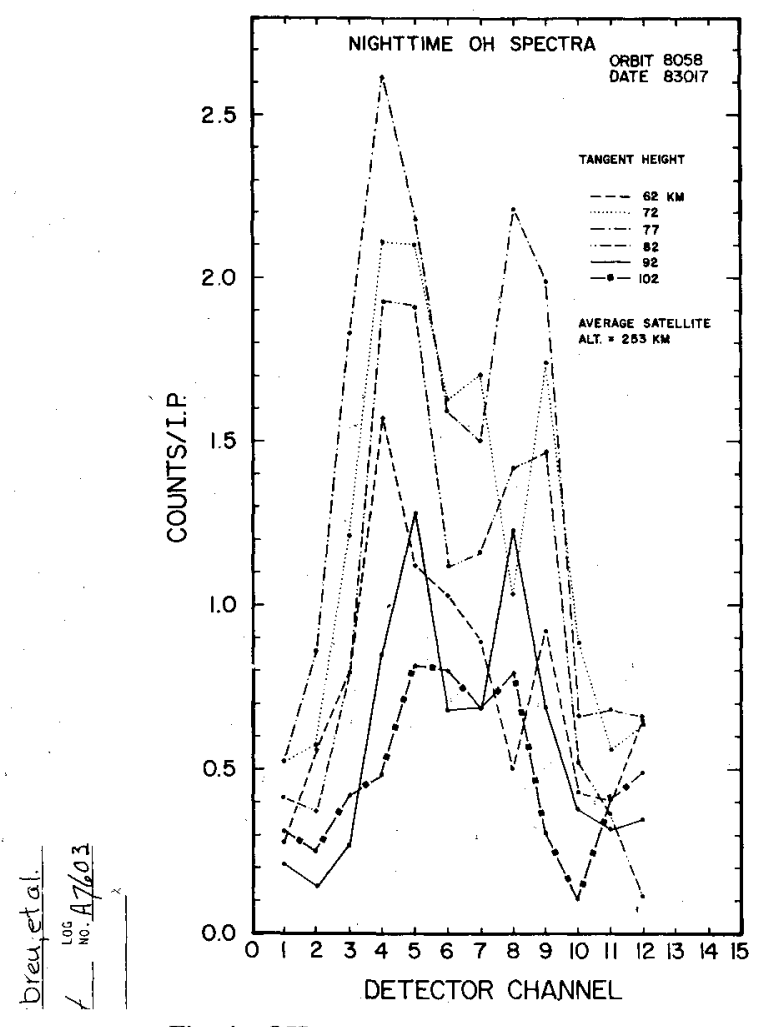

Fig. $4 \mathrm{OH}$ spectra at different tangent heights. identified two lines at 7318.268 and $7318.337 \AA .{ }^{11}$ These two lines would appear four channels apart on the FPI detector, so there is a high probability that these are the two emission lines observed here. The absolute wavelengths of these two lines are not known to the accuracy necessary to predict their relative position to the $\mathrm{O}^{+}\left({ }^{2} \mathrm{P}\right) 7320 \AA$ line on the FPI detector.

The contamination spectrum, normalized to the photometric brightness, is also shown in Fig. 5. The shaded area indicates the statistical uncertainty in the measurements. Since the $\mathrm{OH}$ emission spectrum is doppler shifted by the component of the satellite velocity $(\sim 7.8 \mathrm{~km} / \mathrm{s})$ along the line of sight, we have shifted the contamination spectrum by the same amount for comparison purposes.

The shape of the contaminant spectrum from channels 4 through 12 agrees well with that of the $\mathrm{OH}$ spectrum. This fact lends confidence to the hypothesis that $\mathrm{OH}$ is the contaminant species. The contaminant spectrum, however, suggests an additional peak on channel 2 which is not present on the nightglow $\mathrm{OH}$ spectrum. This could be another $\mathrm{OH}$ line or an emission line from other species, although the relatively narrow bandwidth of the filter $( \pm 10 \AA)$ limits our choices. Since the orbital velocity of the DE satellite imparts about $5 \mathrm{eV}$ relative kinetic energy to the atomic oxygen surface collisions, we do not expect that the atmospheric and atom-surface reactions produce the same $\mathrm{OH}$ vibrational level populations. Vibrationally excited $\mathrm{OH}$ molecules in levels $v \leq 9$ are produced in the atmosphere by the exothermic reaction of $\mathrm{H}$ with $\mathrm{O}_{3}$. Using theoretical calculations Langhoff et al. ${ }^{12}$ computed the vibrational energy level structure for atom-surface reactions and obtained sixteen bound vibrational energy levels. A tabulation of lines in the $7320 \AA( \pm 10 \AA)$ region given by Langhoff et al. ${ }^{12}$, shows a line in the 11-5 band at $7326.8 \AA$ whose strength is comparable to the observed $7318 \AA$ line in the 8-3 band and a weaker line at $7327.0 \AA$ in the 13-6 band. It is possible that the unidentified line present on channel 2 corresponds to one of these transitions. The position of these lines is not known to the accuracy necessary to establish this postulate.

Although we have not proven definitely that $\mathrm{OH}$ is one of the species producing the glow, the data presented here do provide some evidence to this effect. One should be careful not to transfer these results directly to the interpretation of the glow observed in the Shuttle, since different types of contaminants are present in the Shuttle environment.

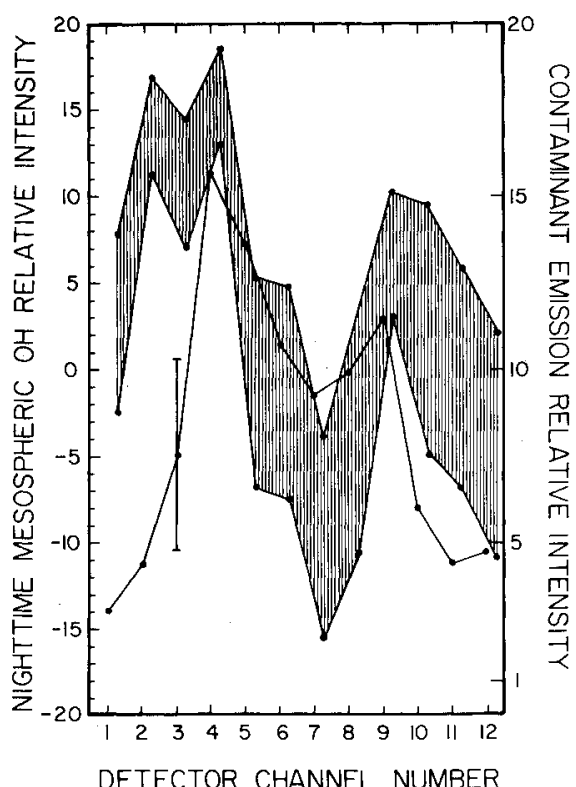

Fig. 5 Comparison of the $\mathrm{OH}$ nightglow and the contaminant glow spectrum (shaded area). 


\section{Conclusion}

Data from the Fabry-Perot interferometer on board the DE-2 satellite have been used to show that the contaminant glow observed by Yee and Abreu at $7320 \AA$ on the AE satellites is produced by emission lines of one of more species. A comparison of the contaminant spectrum near $7320 \AA$ with the nightglow $\mathrm{OH}$ spectrum obtained below $155 \mathrm{~km}$ has provided evidence to the effect that $\mathrm{OH}$ might be one of the species producing the glow.

\section{Acknowledgments}

This work was supported by NASA Grants NAS5-25691 and NAGW-496 to the University of Michigan and by the Air Force Geophysical Laboratory and NSF Grant ATM-8025627 to Harvard University.

\section{References}

${ }^{1}$ Yee, J.H., and Abreu, V.J., "Optical Contamination on the Atmosphere Explorer-E Satellite," Proceedings of SPIE Technical Symposium, 338, 1982, pp. 120-128.

${ }^{2}$ Yee, J.H. and Abreu, V.J., "Visible Glow Induced by SpacecraftEnvironment Interaction." Geophysics Research Letters, 10, 1983, pp. 126-129.

${ }^{3}$ Hays, P.B., Carignan, G.R., Kennedy, B.C., Shepard, G.G., and Walker, J.C.G., "The Visible Airglow Experiment on Atmosphere Explorer," Radio Science, Vol. 8, April 1973, pp. 369-377.
${ }^{4}$ Slanger, T.G., "Conjectures on the Origin of the Surface Glow of Space Vehicles," Geophysical Research Letters, 10, 1983, pp. 130132.

${ }^{5}$ Hays, P.B., Killeen, T. L., and Kennedy, B.C., The Fabry-Perot Interferometer on Dynamics Explorer," Space Science Instrumentation, Vol. 5, No. 4, 1981, pp. 395-416.

${ }^{6}$ Killeen, T.L., Kennedy, B.D., Hays, P.B., Symanow, D.A., and Checkowski, D.H., "An Image Plane Detector for the Fabry-Perot Interferometer on Dynamics Explorer," Applied Optics, 22, 1983, pp. 3503-3513.

${ }^{7}$ Killeen, T.L. and Hays, P.B., "Doppler Line Profile Analysis for a Multi-channel Fabry-Perot Interferometer," Applied Optics, Vol. 23, 1984, pp. 612-620.

${ }^{8}$ Abreu, V.J., Skinner, W.R., and Hays, P.B., "Airglow Measurements of the Variation of the $\mathrm{O}^{+}\left({ }^{2} \mathrm{P}\right)$ Ionization Frequency During Solar Cycle 21," Geophysical Research Letters, 7, 1980, pp. 109-111.

${ }^{9}$ Bates, D.R. and Nicolet, M., "The Photochemistry of Atmospheric Water Vapor, Journal of Geophysical Research, 55, 1950, pp. 301-327.

${ }^{10}$ Watanabe, T., Nakamura, M., and Ogawa, T., "Rocket Measurement of $\mathrm{O}_{2}$ Atmospheric and $\mathrm{OH}$ Meinel Bands in the Airglow, "Journal of Geophysical Research, 86, 1981, pp. 5768-5774.

${ }^{11}$ Coxon, J.A. and Foster, S.C., "Rotational Analysis of Hydroxyl Vibration-Rotation Emission Bands: Molecular Constants for OH $\mathrm{X}^{2} \Pi 1,6 \leq \nu \leq 10$,"' Canadian Journal of Physics, 60, 1982, pp. 41-48.

${ }^{12}$ Langhoff, S.R., Jaffe, R.L., Yee, J.H. and Dalgarno, A., "The Surface Glow of the Atmospheric Explorer-C and E Satellites," Geophysical Research Letters, 10, 1983, pp.896-899.

\section{From the AIAA Progress in Astronautics and Aeronautics Series}

\section{THERMOPHYSICS OF ATMOSPHERIC ENTRY - v. 82}

\section{Edited by T.E. Horton, The University of Mississippi}

Thermophysics denotes a blend of the classical sciences of heat transfer, fluid mechanics, materials, and electromagnetic theory with the microphysical sciences of solid state, physical optics, and atomic and molecular dynamics. All of these sciences are involved and interconnected in the problem of entry into a planetary atmosphere at spaceflight speeds. At such high speeds, the adjacent atmospheric gas is not only compressed and heated to very high temperatures, but stongly reactive, highly radiative, and electronically conductive as well. At the same time, as a consequence of the intense surface heating, the temperature of the material of the entry vehicle is raised to a degree such that material ablation and chemical reaction become prominent. This volume deals with all of these processes, as they are viewed by the research and engineering community today, not only at the detailed physical and chemical level, but also at the system engineering and design level, for spacecraft intended for entry into the atmosphere of the earth and those of other planets. The twenty-two papers in this volume represent some of the most important recent advances in this field, contributed by highly qualified research scientists and engineers with intimate knowlege of current problems.

Published in 1982, 521 pp., $6 \times 9$, illus., $\$ 35.00$ Mem., $\$ 55.00$ List 